\title{
Modeling of non-local interactions on a phase transformation interface
}

\author{
I. Dobovšek ${ }^{1, a}$
}

University of Ljubjana, Faculty of mathematics and physics, Institute of mathematics physics and mechanics, Jadranska 19, Ljubljana 1000, Slovenia.

\begin{abstract}
If an interaction between phases of different composition at an interface is significant, the classical model of an interface is no longer satisfactory. In such cases, the transition layer has to be modeled as a surface phase with intrinsic non-local properties. The boundary of separation, which defines the interface, must possess its own internal structure in order to allow accommodation of complex phenomena while balancing opposite tendencies on each side of the interface. Consequently, an extended model is needed that is capable of describing the intrinsic properties of the interface. To achieve this goal, we employ a special model of an interface with simple structure, where the mathematical notion of a simple structure corresponds to the material model without rotational degrees of freedom in each lattice point of the interface. By employing characteristic features of the model, we are in a position to describe quantitatively and qualitatively the structural and compositional non-local changes across the interface.
\end{abstract}

\section{Introduction}

Many of the properties of polycrystalline materials are determined to a large extent by the properties of their internal interfaces. The study of polycrystalline materials can be often considered as the study of their interfaces. Since the properties of interfaces between crystals depend on their structure, analysis of the structure and behavior of interfaces has become a very important research area in the larger field of science as well as in engineering modeling of materials. Interfacial phenomena occur whenever a continuum is present that can exist in at least two different physical states and there is some mechanism that generates or enforces a spatial separation between these states. Such localized transitions between different phases usually exhibit intrinsic structure over the finite width. Interfaces often exist in polycrystalline multiphase materials in a large number of configurations including those with curved structure. The simplest interface is a single isolated planar interface separating two otherwise perfect crystals of the same, or different crystalline phases, i.e., a planar interface in a bicrystal. The concept of an ideal bicrystal containing a planar interface serves as a prototype for understanding the nonlocal properties of interfaces. Geometric description of a bicrystal consists of characterizing the relationship between the crystals on both sides of the interface and the interface plane where the transition between one phase and another within the bulk of the crystal generally takes place over a zone of finite width, which, as in some standard approaches after employing the corresponding limiting procedure, can shrink to zero. For such a system, kinematics and mechanics of behavior can be established successfully, though development of kinematics is more complicated than in the standard continuum mechanics. In theories that have been developed so far, the interface between phases was usually considered sharp. However, if an interaction

\footnotetext{
a e-mail: igor.dobovsek@fmf.uni-lj.si
} 
between volume phases of a bicrystal and an interface is significant, the classical model of an interface as a singular surface with the standard jump conditions complementing the balance field equations of the volume phase is no longer satisfactory. In such cases, the transition layer has to be modeled as a surface phase with intrinsic non-local properties. The boundary of separation, which defines the interface, must possess its own internal structure in order to allow accommodation of complex phenomena while balancing opposite tendencies on each side of the interface. Consequently, an extended model is needed that is capable of describing the intrinsic properties of the interface. To achieve this goal, we employ a special model of an interface with simple structure, where the mathematical notion of a simple structure corresponds to the material model without rotational degrees of freedom in each lattice point of the interface. By employing characteristic features of the model, we are in a position to describe quantitatively and qualitatively the non-local structural and compositional changes across the interface.

\section{Classification of interfaces}

There are various ways of classifying solid-solid interfaces. An interface between two crystals of the same phase with an orientation difference or a translation between two crystals of the same phase across an interface is referred to as a homophase interface. Examples of homophase interfaces are grain boundaries, twin boundaries, stacking faults. On the other hand, the heterophase interface designates an interface between two crystals of different phase. Crystals differ in composition, Bravais lattice or both. Heterophase class of interfaces can be further divided according to the degree of atomic matching or coherency across the interface into the following categories: A) Fully coherent interface (unrelaxed), where there is complete continuity of atomic planes and lines across the interface between the two phases; B) Semi coherent interface (relaxed) in which the disregistry between two crystal structures across the interface is accommodated by periodic misfit dislocations in the interface; C) Incoherent interface, where atomic matching is sufficiently poor so there is no correspondence of atom planes and lines across the interface even locally. Another possible classification of interfaces is regarding the strength of interaction and the long or short range order. Commensurate interfaces are fully coherent with long-range order, while incommensurate interface exhibit no long-range order and are incoherent. A category in between is the so-called family of incommensurate interfaces with semi coherent interfacial structure.

In principle any phase transformation front should be perceived as an interphase boundary between the two phases of different composition and its own structure, where one of the phases represents a parent phase and the other a product phase. Since each evolving phase like martensite and austenite in the process of phase transformation exhibits its own intrinsic properties, which may differ in crystal structure, lattice constant and chemical composition, the interface that separates both phases is at least to some extent influenced by long range interaction between both evolving phases in the bulk. Such an interaction culminates on the interface as interplay between both phases.

\section{Self energy of an interface}

Theoretical origins of non-local structure and interaction of complex phenomena taking place on an interface can be recognized and derived from first principles and ab-initio approach. Certain parts of theoretical development have been successfully employed in the past in connection with atomistic and phenomenological modeling of epitaxial growth [8],[19], [29]. We use similar ideas from the cited literature in order to establish necessary background for understanding physics of the problem and to justify particular choices that have been made during development of phenomenological model. As a starting point in derivation, we define the energy of a binary system including the separation boundary - an interface. It is assumed that the total energy of 


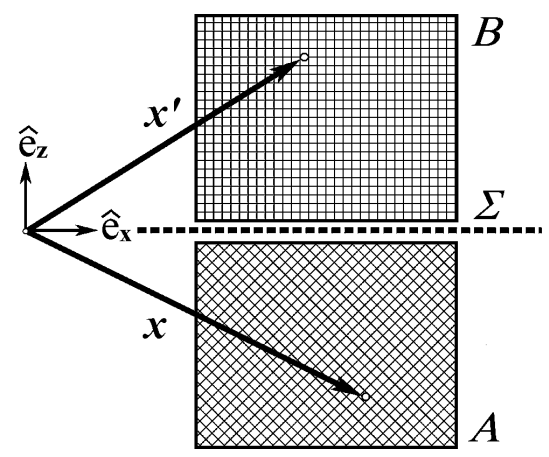

Fig. 1. Thin film interface $\Sigma$ in heterophase system between phases $A$ and $B$.

the atomic assembly can be represented by a sum of pair interactions in the following form

$$
E_{T}=\sum_{i}^{A} \sum_{j}^{B} \mathcal{U}_{A B}\left(\boldsymbol{x}_{i}-\boldsymbol{x}_{j}^{\prime}\right)+\frac{1}{2} \sum_{i \neq j}^{A} \mathcal{U}_{A A}\left(\boldsymbol{x}_{i}-\boldsymbol{x}_{j}\right)+\frac{1}{2} \sum_{i \neq j}^{B} \mathcal{U}_{B B}\left(\boldsymbol{x}_{i}^{\prime}-\boldsymbol{x}_{j}^{\prime}\right),
$$

where the last two terms represent the self-energy of both phases. Here for the sake of generality we use the notation $A$ and $B$ to distinguish between both phases. The important part of the expression is the first term, which designates an interaction potential between atoms in phase $A$ and phase $B$, and gives a description of an interaction which takes place on an interface. The expression describes the ground state energy of the system and is defined with respect to the undeformed configuration

$$
\boldsymbol{x} \equiv \boldsymbol{x}_{0}, \quad \boldsymbol{x}^{\prime} \equiv \boldsymbol{x}_{0}^{\prime}
$$

of the system where $\boldsymbol{x} \in A$ and $\boldsymbol{x}^{\prime} \in B$ designate the atomic positions of semi-infinite parts $A$ and $B$ of the crystal separated by the interface $\Sigma$. Under the action of external disturbance, the atoms near the interface change their placement from undistorted ground state to deformed configuration by means of two displacement vectors from each side of the surface

$$
\boldsymbol{x}_{i}=\boldsymbol{x}_{i}^{0}+\boldsymbol{u}_{i}\left(\boldsymbol{x}_{i}^{0}\right), \quad \boldsymbol{x}_{i}^{\prime}=\boldsymbol{x}_{i}^{0^{\prime}}+\boldsymbol{u}_{i}^{\prime}\left(\boldsymbol{x}_{i}^{0^{\prime}}\right)
$$

so that the self-energy of the system in deformed configuration can be written formally as

$$
E_{T}=E_{A}^{0}+E_{B}^{0}+E_{A}+E_{B}+\sum_{i}^{A} \sum_{j}^{B} \mathcal{U}_{A B}\left(\boldsymbol{x}_{i}^{0}+\boldsymbol{u}_{i}-\boldsymbol{x}_{j}^{0^{\prime}}-\boldsymbol{u}_{j}{ }^{\prime}\right),
$$

where $E_{A}^{0}$ and $E_{B}^{0}$ are the self-energies of both undistorted semi crystals in undistorted phase. These two terms are not important from the point of view of further development and can be considered as additive constants in the expression of total self-energy of the system. On the other hand, $E_{A}$ and $E_{B}$ represent self-energies of both semi crystals each in different phase, a parent and a product phase. The structure of particular part of former expression, which describes the interaction on the interface between atoms from different phases, i.e., $\mathcal{U}_{A B}$, clearly shows a non-local nature of interaction since the functional part of the expression depends on the difference between relative positions of the atoms from each separate phase in the close vicinity of the interface. The length scale of separation zone is small, but not infinitesimal. It is of reasonably short range and extends over only a few atomic layers on either side of the interface. It can provide a characteristic length scale needed for construction of corresponding non-local phenomenological continuum model.

\section{The mathematical model}

Since interface phenomena occur whenever a continuum is present, that can exist in at least two different physical states and there is some mechanism that generates or enforces a spatial 


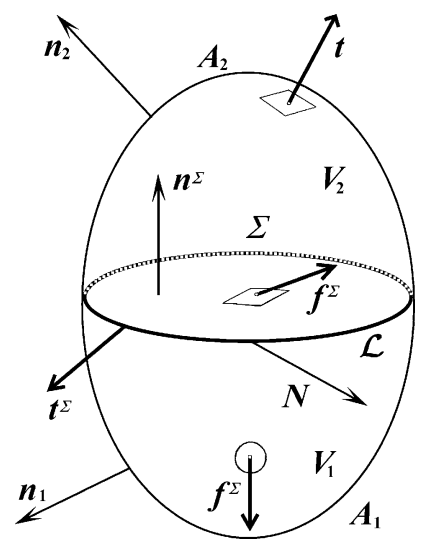

Fig. 2. Geometry of a two-phase system. $\Sigma$ represents an interface of the system with intrinsic structure - surface density, surface force, interface traction; $\Sigma:\left\{\rho_{\Sigma}, \boldsymbol{f}^{\Sigma}, \boldsymbol{t}^{\Sigma}\right\}$.

separation between these states, like martensite and austenite, a mathematical model of a two phase continuum with a moving interface serves as a starting point in theoretical modeling of the evolution of a phase transformation front [4], [5]. The main purpose of the paper is to establish a phenomenological model of a heterophase interface $\Sigma(t)$ evolving in time with velocity $\boldsymbol{v}^{\Sigma}$ that can sustain surface stresses $\boldsymbol{\sigma}^{\Sigma}$ between both phases, surface forces $\boldsymbol{f}^{\Sigma}$ defined per unit mass of the interface with its own non-zero surface density $\rho_{\Sigma}$.

Consider a material body in space-time $(\mathcal{B}, t) \subset \mathbb{R}^{3} \times \mathbb{R}_{+}$defined on a set $\{(\boldsymbol{x}, t): \boldsymbol{x} \in$ $\left.\mathbb{R}^{3}, t \in[0, \infty)\right\}$, where a material point in $\mathcal{B}$ is determined by its position vector $\boldsymbol{x}$ relative to the fixed Cartesian coordinate system with the orthonormal basis $\left\{\widehat{\mathrm{e}}_{1}, \widehat{\mathrm{e}}_{2}, \widehat{\mathrm{e}}_{3}\right\}, \widehat{\mathrm{e}}_{i} \cdot \widehat{\mathrm{e}}_{j}=\delta_{i j}$. The symbol $\delta_{i j}$ designates the Kronecker's delta and the summation convention over repeated indices is implied in subsequent expressions. An evolving two-phase region $\left\{\mathcal{B} \equiv V(t): V_{1}(t), V_{2}(t)\right\}$ in $\mathbb{R}^{3}$ is a time-dependent region $\mathcal{B} \equiv V(t) \subset \mathbb{R}^{3}$ representing the volume of the bulk, together with closed sub-regions $V_{1}(t)$ and $V_{2}(t)$ occupied by both phases such that $V=V_{1} \cup V_{2}$. The intersection $\Sigma(t)=V_{1} \cap V_{2}$ is a smoothly evolving surface - an interface with the normal $\boldsymbol{n}^{\Sigma}$ pointing from $V_{1}$ to $V_{2}, A_{i}$ and $\boldsymbol{n}_{i}$ are surfaces and corresponding outer normals of both phases $(i=1,2), \mathcal{L}=\Sigma \cap\left\{A_{1} \cup A_{2}\right\}$ is the contact line, $\boldsymbol{N}$ is the normal to $\mathcal{L}$ on $\Sigma, \boldsymbol{v}_{i}$ is the velocity of the material point in $V_{i}, \boldsymbol{v}^{\Sigma}$ is the velocity of the interface. We define $\llbracket \cdot \rrbracket=(\cdot)_{1}-(\cdot)_{2}$ as a standard jump operator across the interface. Terms designated with the subscript or superscript $(\cdot)_{\Sigma}$ pertain to the quantities associated with the surface of the interface. Figure 1 illustrates geometry and applied loads on the system.

\subsection{Mass and interface mass density}

Because we are dealing with a body consisting of two phases separated by an interface, we define the mass associated with the volume of the bulk as well as surface mass associated with dividing the surface of the system, together with the corresponding densities. So for mass and surface mass defined on evolving time-dependent sets $\left(V_{t}, \Sigma_{t}\right)$ we have for the bulk

$$
M^{(3)}=\int_{V_{t}} \rho d V, \quad \rho=d M^{(3)} / d V
$$

and for the interface

$$
M^{(2)}=\int_{\Sigma_{t}} \rho_{\Sigma} d \Sigma, \quad \rho_{\Sigma}=d M^{(2)} / d \Sigma .
$$

With this definitions, the total mass of the body $\mathcal{B}$ on $\left\{V_{1} \cup V_{2} \cup \Sigma\right\}$ can be calculated as

$$
M=\int_{V_{1}} \rho_{1} d V+\int_{V_{2}} \rho_{2} d V+\int_{\Sigma_{t}} \rho_{\Sigma} d \Sigma .
$$




\subsection{Spatial and surface stresses}

The acting forces on a body are of two different origins. The body forces are related to the mass of the body of each particular phase and act directly on a material particle. If measured per unit mass, mass forces $\boldsymbol{F}_{M}$ can be defined as

$$
\boldsymbol{f}=\frac{d \boldsymbol{F}_{M}}{d M}
$$

Contact loads belong to another category of forces that are exerted on a body acting on a common surface of contact. Measured per unit area, vectors of contact surface forces $\boldsymbol{F}_{A}$ are calculated as

$$
\boldsymbol{t}(\boldsymbol{n})=\frac{d \boldsymbol{F}_{A}}{d A}
$$

The corresponding vectors of stress $\boldsymbol{t}$ are connected with tensors of stress $\boldsymbol{\sigma}$ via Cauchy's stress principle

$$
\boldsymbol{t}(\boldsymbol{n})=\boldsymbol{n} \cdot \boldsymbol{\sigma},
$$

where $\boldsymbol{n}$ is a surface normal on $\mathcal{B}$. On a surface of interface we postulate the existence of both categories of forces, namely the family of body forces and the family of contact forces. From the first category, the surface mass forces $\boldsymbol{F}_{M \Sigma}$ acting on the interface $\Sigma$ bounded by a contact curve $\mathcal{L}$, can be calculated as

$$
\boldsymbol{f}^{\Sigma}=\frac{d \boldsymbol{F}_{M \Sigma}}{d M_{\Sigma}}
$$

The category of contact forces is represented by a vector of line forces $\boldsymbol{F}_{L}$ acting on a boundary curve $\mathcal{L}$ with a normal $N$

$$
\boldsymbol{t}^{\Sigma}(\boldsymbol{N})=\frac{d \boldsymbol{F}_{L}}{d L}
$$

which can be associated with surface stress tensor $\boldsymbol{\sigma}^{\Sigma}$ of the interface by using a relation

$$
t^{\Sigma}(N)=N \cdot \sigma^{\Sigma}
$$

\section{Balance of Linear Momentum}

For the two-phase material continuum $V=V_{1} \cup V_{2}, A=A_{1} \cup A_{2} \cup \Sigma$ the balance of linear momentum in a global form can be written as

$$
\begin{gathered}
\frac{d}{d t}\left(\int_{V_{1}} \rho_{1} \boldsymbol{v}_{1} d V+\int_{V_{2}} \rho_{2} \boldsymbol{v}_{2} d V+\int_{\Sigma} \rho_{\Sigma} \boldsymbol{v}^{\Sigma} d \Sigma\right)=\int_{V_{1}} \rho_{1} \boldsymbol{f}_{1} d V+\int_{V_{2}} \rho_{2} \boldsymbol{f}_{2} d V \\
+\int_{\Sigma} \rho_{\Sigma} \boldsymbol{f}_{\Sigma} d \Sigma+\int_{A_{1}} \boldsymbol{n}_{1} \cdot \boldsymbol{\sigma}_{1} d A+\int_{A_{2}} \boldsymbol{n}_{2} \cdot \boldsymbol{\sigma}_{2} d A+\int_{\mathcal{L}} \boldsymbol{N} \cdot \boldsymbol{\sigma}^{\Sigma} d \mathcal{L} .
\end{gathered}
$$

Due to the fact that for each of separate phases $V_{i}(i=1,2)$ the following balance equation must be satisfied

$$
\frac{d}{d t} \int_{V_{i}} \rho_{i} \boldsymbol{v}_{i} d V=\int_{V_{i}} \rho_{i} \boldsymbol{f}_{i} d V+\int_{A_{i}} \boldsymbol{n}_{i} \cdot \boldsymbol{\sigma}_{i} d A+\int_{\Sigma} \boldsymbol{n}_{i} \cdot \boldsymbol{\sigma}_{i} d \Sigma-\int_{\Sigma} \rho_{i} \boldsymbol{v}_{i}\left(\boldsymbol{v}_{i}-\boldsymbol{v}^{\Sigma}\right) \cdot \boldsymbol{n}_{i} d \Sigma,
$$

we can derive the weak form of the equations of balance. Equations of momentum balance for both phases in bulk follow directly from Eq. (15). Here we give only a final expression for the interface. The interface momentum balance in the weak form reads

$$
\begin{aligned}
\frac{d}{d t} \int_{\Sigma} \rho_{\Sigma} \boldsymbol{v}^{\Sigma} d \Sigma & =\int_{\mathcal{L}} \boldsymbol{N} \cdot \boldsymbol{\sigma}^{\Sigma} d \mathcal{L}+\int_{\Sigma} \rho_{\Sigma} \boldsymbol{f}^{\Sigma} d \Sigma-\int_{\Sigma}\left(\boldsymbol{n}_{1} \cdot \boldsymbol{\sigma}_{1}+\boldsymbol{n}_{2} \cdot \boldsymbol{\sigma}_{2}\right) d \Sigma \\
& +\int_{\Sigma}\left[\rho_{1} \boldsymbol{v}_{1} \otimes\left(\boldsymbol{v}_{1}-\boldsymbol{v}^{\Sigma}\right) \cdot \boldsymbol{n}_{1}+\rho_{2} \boldsymbol{v}_{2} \otimes\left(\boldsymbol{v}_{2}-\boldsymbol{v}^{\Sigma}\right) \cdot \boldsymbol{n}_{2}\right] d \Sigma
\end{aligned}
$$


After using the surface divergence theorem together with the interface mass balance equation, details of derivation can be found in [28], the corresponding strong form of equations of linear momentum for the interface follows directly as

$$
\rho_{\Sigma} \frac{d \boldsymbol{v}^{\Sigma}}{d t}=\nabla_{\Sigma} \cdot \boldsymbol{\sigma}^{\Sigma}+\rho_{\Sigma} \boldsymbol{f}^{\Sigma}-\boldsymbol{n} \cdot \llbracket \boldsymbol{\sigma} \rrbracket+\llbracket \rho\left(\boldsymbol{v}-\boldsymbol{v}^{\Sigma}\right) \otimes\left(\boldsymbol{v}-\boldsymbol{v}^{\Sigma}\right) \rrbracket \cdot \boldsymbol{n},
$$

where we have taken that $\boldsymbol{n}=\boldsymbol{n}_{1}=-\boldsymbol{n}_{2}=\boldsymbol{n}^{\Sigma}$ holds on the interface, according to the definitions at the beginning of the chapter. For smooth fields acting on a surface of interface without jumps in traction and kinetic terms we have

$$
\boldsymbol{n} \cdot \llbracket \boldsymbol{\sigma} \rrbracket=\mathbf{0}, \quad \llbracket \rho\left(\boldsymbol{v}-\boldsymbol{v}^{\Sigma}\right) \otimes\left(\boldsymbol{v}-\boldsymbol{v}^{\Sigma}\right) \rrbracket \cdot \boldsymbol{n}=\mathbf{0}
$$

and Eq. (17) takes on a standard form of momentum balance, but with all entities referring to the surface of the interface

$$
\rho_{\Sigma} \frac{d \boldsymbol{v}^{\Sigma}}{d t}=\nabla_{\Sigma} \cdot \boldsymbol{\sigma}^{\Sigma}+\rho_{\Sigma} \boldsymbol{f}^{\Sigma}
$$

\section{Decomposition of strain}

The local elastic small strain in the bulk is defined in a standard way as

$$
\boldsymbol{\epsilon}=\operatorname{sym}[\nabla \boldsymbol{u}(\boldsymbol{x})], \quad \epsilon_{i j}(\boldsymbol{x})=\frac{1}{2}\left(\frac{\partial u_{i}(\boldsymbol{x})}{\partial x_{j}}+\frac{\partial u_{j}(\boldsymbol{x})}{\partial x_{i}}\right),
$$

and provides a possible link between continuum and microstructural structure embedded in the phenomenological concept of interfacial energy. Since displacement fields are continuous across the interface, continuity of local kinematics variables, but not their gradients, allows decomposition of strain $\boldsymbol{\epsilon}$ in two complementary parts: interior $\boldsymbol{\epsilon}^{\Sigma}$ and exterior $\boldsymbol{\epsilon}^{N}$. Interior part of decomposition maps the local spatial fields onto the plane of interface $\Sigma$ with normal $\boldsymbol{n}^{\Sigma}$, while exterior part projects the bulk strain in the direction of the normal $\boldsymbol{n}^{\Sigma}$ out of the interface plane

$$
\epsilon=\epsilon^{\Sigma}+\epsilon^{N}
$$

With the help of projection operator

$$
\mathrm{P}^{\Sigma}=\boldsymbol{I}-\boldsymbol{n}^{\Sigma} \otimes \boldsymbol{n}^{\Sigma}=\left(\delta_{i j}-n_{i}^{\Sigma} n_{j}^{\Sigma}\right) \widehat{\mathrm{e}}_{i} \otimes \widehat{\mathrm{e}}_{j},
$$

the interior part of decomposition representing the surface strain can be written as

$$
\boldsymbol{\epsilon}^{\Sigma}=\mathrm{P}^{\Sigma} \cdot \boldsymbol{\epsilon} \cdot \mathrm{P}^{\Sigma}=\left(\mathrm{P}_{i k}^{\Sigma} \epsilon_{k l} \mathrm{P}_{l j}^{\Sigma}\right) \widehat{\mathrm{e}}_{i} \otimes \widehat{\mathrm{e}}_{j} .
$$

The outer part of decomposition is achieved by introducing an operator

$$
\begin{gathered}
\mathcal{P}^{N}=\frac{1}{2}\left(n_{i}^{\Sigma} n_{k}^{\Sigma} \delta_{j l}+n_{j}^{\Sigma} n_{l}^{\Sigma} \delta_{i k}+n_{j}^{\Sigma} n_{k}^{\Sigma} \delta_{i l}+n_{i}^{\Sigma} n_{l}^{\Sigma} \delta_{j k}\right. \\
\left.-n_{i}^{\Sigma} n_{j}^{\Sigma} n_{k}^{\Sigma} n_{l}^{\Sigma}\right) \widehat{\mathrm{e}}_{i} \otimes \widehat{\mathrm{e}}_{j} \otimes \widehat{\mathrm{e}}_{k} \otimes \widehat{\mathrm{e}}_{l},
\end{gathered}
$$

so that the normal out of plane strain can be calculated by using a transformation

$$
\epsilon^{N}=\mathcal{P}^{N}: \epsilon=\left(\mathcal{P}_{i j k l}^{N} \epsilon_{k l}\right) \widehat{\mathrm{e}}_{i} \otimes \widehat{\mathrm{e}}_{j} .
$$

Such decomposition is useful because it provides a natural way of connecting the surface strain with surface stress which is essential part of constructing the corresponding constitutive law for the interface. In practical applications we usually employ an intrinsic coordinate system where components of the normal vector $\boldsymbol{n}^{\Sigma}$ are $\left(n_{1}, n_{2}, n_{3}\right)=(0,0,1)$. For this system the inner and outer terms are just

$$
\boldsymbol{\epsilon}^{\Sigma}=\left[\begin{array}{ccc}
\epsilon_{11} & \epsilon_{12} & 0 \\
\epsilon_{12} & \epsilon_{22} & 0 \\
0 & 0 & 0
\end{array}\right], \quad \boldsymbol{\epsilon}^{N}=\left[\begin{array}{ccc}
0 & 0 & \epsilon_{13} \\
0 & 0 & \epsilon_{23} \\
\epsilon_{13} & \epsilon_{23} & \epsilon_{33}
\end{array}\right] \text {. }
$$




\section{Non-local structure of constitutive relation}

If we look back at terms which describe self energy of each individual phase Eq. (4), the variations of the energy away from the interface describe what is essentially local elastic behavior, which can be approximated with continuum elasticity theory to a large degree of accuracy. Therefore, during development of the model of an interface with intrinsic structure, we consider the case where interface is embedded in a homogeneous, elastic crystal. In such a form the vicinity of the interface retains intrinsic local structure. On the other hand, the self-energy of the interface is assumed to exhibit a long range order of interaction, and as such can be perceived as non-local. Hence, the surrounding medium deforms locally, but surface entities are mathematically described in the classical form of non-local elasticity. Our goal is to establish a constitutive relation for the interface of the form

$$
\boldsymbol{\sigma}^{\Sigma}=\boldsymbol{\sigma}^{\Sigma}\left(\boldsymbol{\epsilon}^{\Sigma}\right), \quad \boldsymbol{\sigma}^{\Sigma}=\sigma_{a b}^{\Sigma}\left(\epsilon_{c d}^{\Sigma}\right) \widehat{\mathrm{e}}_{a} \otimes \widehat{\mathrm{e}}_{b}, \quad\{(a, b, c, d) \in(1,2)\}
$$

that would incorporate the most important intrinsic features of a thin film separating two phases of different composition with some degree of non-local behavior.

\subsection{Surface tension elasticity}

Surface and interface stresses can significantly modify the structure and mechanical behavior of materials if the characteristic length scale is reduced to the nanometer range. Surface stress effects are important in materials with high density of surfaces and interfaces. Particularly in nanostructured materials where there is a significant difference in lattice parameters between bulk materials. The existing surface stress and structural mismatch at an interface can lead to significant variations in physical properties. From the perspective of mechanics, such variations are important because of the changes in magnitude of elastic modulus, which can be in the range of several percent. It is widely accepted that this change comes from the contribution of the higher order terms in the expansion of interatomic potential with respect to the atomic distance, which can be associated with surface phenomena or the so-called surface modulus of elasticity. The smaller the length scale, the more significant is the effect on the overall mechanical behavior of the material.

For such systems, the total energy in continuum approach is written as the sum of the energy of the bulk B and the surface energy $\Gamma$

$$
E_{T}=\mathrm{B}+\Gamma=\int_{\mathcal{B}} W(\boldsymbol{\epsilon}) d V+\int_{\Sigma} \gamma\left(\boldsymbol{n}^{\Sigma}\right) d A
$$

$W$ is the strain energy density accounting for the energy of bulk deformation, while the second term defines the amount of surface energy density $\gamma$ which obviously depends on the global orientation of the interface $\Sigma$ defined by its normal $\boldsymbol{n}^{\Sigma}$. This particular form of the total energy has been extensively treated in [12] and [13]. For the particular choice of surface energy of the form

$$
\Gamma=\frac{1}{2} \epsilon^{\Sigma}: \mathbf{C}^{\Sigma}: \epsilon^{\Sigma}=\frac{1}{2} \epsilon_{a b}^{\Sigma} C_{a b c d}^{\Sigma} \epsilon_{c d}^{\Sigma},
$$

where the surface elasticity tensor under the complete isotropy group of transformations is defined as

$$
C_{a b c d}^{\Sigma}=\lambda^{\Sigma} \delta_{a b} \delta_{c d}+\mu^{\Sigma}\left(\delta_{a c} \delta_{b d}+\delta_{b c} \delta_{a d}\right), \quad\{(a, b, c, d) \in(1,2)\}
$$

leads to a special form of the flat surface tension elasticity theory where constitutive parameters $\lambda^{\Sigma}$ and $\mu^{\Sigma}$ can be directly related to the term $\Gamma$ describing the surface energy of the interface. The model allows incorporation of surface tension, which can be directly associated with the constitutive parameter $\lambda^{\Sigma}$. Further theoretical details are given in [13], while exhaustive experimental data for different systems can be found in [2]. 


\subsection{Non-local elasticity}

Another model that provides additional possibilities in modeling of complex non-local phenomena on an interface is given in integral form [7] and it carries a very close resemblance with the structure of ab-initio approach, since it can be directly connected with the lattice theory. In the non-local field theory it is assumed that the stress at a material point $\boldsymbol{x}$ depends not only on strains at $\boldsymbol{x}$, but also on strains at $\boldsymbol{x}^{\prime}$ in the close vicinity of the same point. The domain of influence depends on the magnitude of the long-range order, but in principle it extends through a few atomic distances at least. Local theories neglect this effect completely, but in non-local elasticity the constitutive equation between stress and strain is defined as

$$
\sigma_{i j}(\boldsymbol{x})=\int_{\mathcal{B}} C_{i j k l}\left(\boldsymbol{x}-\boldsymbol{x}^{\prime}\right) \epsilon_{k l}\left(\boldsymbol{x}^{\prime}\right) d \Omega\left(\boldsymbol{x}^{\prime}\right)
$$

Such a relation takes into account non-local interaction between material points where the expression $C_{i j k l}\left(\boldsymbol{x}-\boldsymbol{x}^{\prime}\right)$ represents the tensor of non-local elastic moduli. For homogeneous non-local elastic medium, the range of interaction between material points depends only on a relative distance between material points, so that

$$
C_{i j k l}\left(\boldsymbol{x}-\boldsymbol{x}^{\prime}\right)=C_{i j k l}\left(\left|\boldsymbol{x}-\boldsymbol{x}^{\prime}\right|\right)
$$

A variety of other formulations are possible and can be found in the literature [7]. For an elastic material with the standard isotropic properties we can take

$$
C_{i j k l}=\lambda\left(\boldsymbol{x}-\boldsymbol{x}^{\prime}\right) \delta_{i j} \delta_{k l}+\mu\left(\boldsymbol{x}-\boldsymbol{x}^{\prime}\right)\left(\delta_{i k} \delta_{j l}+\delta_{j k} \delta_{i l}\right) .
$$

Here $\lambda\left(\boldsymbol{x}-\boldsymbol{x}^{\prime}\right)$ and $\mu\left(\boldsymbol{x}-\boldsymbol{x}^{\prime}\right)$ denote the corresponding non-local Lamé's constants. For more general case of anisotropic constitutive response, the symmetry structure of the constitutive tensor can be adjusted accordingly. An effective application of the theory can be accomplished by utilizing the idea of attenuation function kernel where $\alpha\left(\left|\boldsymbol{x}^{\prime}-\boldsymbol{x}\right|\right)$ represents an influence function, so that in view of Eqs. (30), (31) and 32) the constitutive relation takes on the form of integral relationship

$$
\sigma_{i j}(\boldsymbol{x})=\int_{\mathcal{B}} \alpha\left(\left|\boldsymbol{x}^{\prime}-\boldsymbol{x}\right|, \ell\right)\left[\lambda \delta_{i j} \delta_{k l}+\mu\left(\delta_{i k} \delta_{j l}+\delta_{j k} \delta_{i l}\right)\right] \epsilon_{k l}\left(\boldsymbol{x}^{\prime}\right) d \Omega\left(\boldsymbol{x}^{\prime}\right)
$$

in which $\lambda$ and $\mu$ are the usual Lamé's constants. The kernel $\alpha\left(\left|\boldsymbol{x}^{\prime}-\boldsymbol{x}\right|, \ell\right)$ is decreasing with the distance from $\boldsymbol{x}$, and as a non-local distance function depends on a normalized length scale $\ell$. This is an internal parameter of characteristic normalized length and represents an intrinsic property of a material. In majority of cases it is appropriate to consider $\ell$ as a multiple of a lattice parameter $a$, i.e., $\ell=n a / L$, normalized with an external characteristic length $L$. In our case it may represent the width of the interface $\Sigma$ or some other characteristic feature of the material. In the limit as $\ell \rightarrow 0$ Eq. (33) must transform to the classical constitutive relation of linear elasticity. This implies that the kernel $\alpha$ consists of a Dirac delta sequence

$$
\lim _{\ell \rightarrow 0} \alpha\left(\left|\boldsymbol{x}^{\prime}-\boldsymbol{x}\right|, \ell\right)=\delta\left(\left|\boldsymbol{x}^{\prime}-\boldsymbol{x}\right|\right)
$$

For small internal characteristic length, when $\ell \rightarrow 1$, non-local theory transforms into the discrete lattice theory. Different representations of the kernel $\alpha(|\boldsymbol{x}|, \ell)$ are possible. According to [7], certain choices of kernel function $\alpha(|\boldsymbol{x}|, \ell)$ with particular mathematical structure yield good matching between phonon dispersion curves and the Born-Karman model of the atomic lattice dynamics and provide very good approximation for non-local moduli. Significant progress has been made for calibrating non-local characteristics from atomic dispersion curves for two dimensional lattices. The results of such studies are directly applicable in development of the non-local model of interface with intrinsic structure, the work which is currently in progress. 


\section{Concluding remarks}

Interfacial systems are extremely complicated from experimental as well as theoretical aspect of modeling. In real materials such systems exhibit physical properties and characteristics in chemical composition that differ significantly from the surrounding material in the bulk. Due to strong intrinsic inhomogeneity and different features of geometrical structure, namely the macroscopic, microscopic and atomic, it has become apparent that classical approach in modeling is not adequate to capture the most important attributes of such systems. Consequently, in addition to macroscopic degrees of freedom, the model has to contain internal microscopic degrees of freedom associated with corresponding parameters chosen in a manner that allows an adequate description of intrinsic features of an interface. In this work the emphasis was made on incorporation of a length scale into macroscopic field equations by using well-developed structure of non-local field theories expressed in terms of integral formulation of non-local elasticity. Even in its simplest form, the characteristic features of non-local theory of elasticity can provide new possibilities in modeling the correlation effects among the macroscopic, microscopic and atomic structure that take place on an interface with intrinsic complex morphology and composition.

\section{References}

1. V. S. Boyko, R. I. Garber, A. M. Kossevich, Reversible Crystal Plasticity, AIP Press, New York, 1997.

2. R. C. Cammarata, Progress in Surface Science, 46, (1994) 1-38.

3. M. Cherkaoui, M. Berveiller, Arch. Appl. Mech. 70, (2000) 159-181.

4. I. Dobovšek, Z. angew. Math. Mech., 79-S2,(1999) s405-406.

5. I. Dobovšek, Materials Science and Engineering A, 481-482,(2008) 356-361.

6. E. Kröner, Arch. Rat. Mech. Anal., 4, (1960) 273-334.

7. A. C. Eringen, J. Appl. Phys., 54,(1983) 4703-4710.

8. N. H. Fletcher and K. W. Lodge, Epitaxial Growth, Part B, J. W. Matthews ed., Academic Press, New York, 529-557, 1975.

9. G. M. C. Fisher and M. J. Leitman, Arch. Ration. Mech. and Anal., 30, (1968) 225-262.

10. F. D. Fischer, E. R.Oberaigner, Arch. Appl. Mech. 71, (2001) 193-205.

11. A. E. Green, P. M. Naghdi and W. L. Wainwright Arch. Ration. Mech. and Anal., 20, (1965) 287-308.

12. M. E. Gurtin, Thermomechanics of Evolving Phase Boundaries in the Plane, Clarendon Press, Oxford, 1993

13. M. E. Gurtin and I. Murdoch, Arch. Ration. Mech. and Anal., 57, (1975) 291-323.

14. Y. Ida, J. Geophys. Res., 74, (1969) 3208-3218.

15. A. G. Khachaturian, Theory of Structural Transformations in Solids, J. Wiley \& Sons, 1983.

16. N. Liosatos, A. E. Romanov, M. Zaiser, and E. C. Aifantis, Scripta Materialia, 38, (1998) 819-826.

17. W. Lojkowski and H. J. Fecht, Prog. Mat. Sci., 45, (2000) 339-568.

18. J. W. Mathews, Epitaxial Growth. Part B, J. W. Matthews ed., Academic Press, 1975, 559-609.

19. J. H. van der Merwe and C. A. B. Ball, Epitaxial Growth, Part B, J. W. Matthews ed., Academic Press, New York, 1975, 493-528.

20. G. P. Moeckel, Arch. Ration. Mech. and Anal., 57, (1975) 255-280.

21. L. G. Napolitano, Acta Astronautica, 6, (1979) 1093-1112.

22. Z. Nishiyama, Martensitic Transformation, Academic Press, New York, 1978.

23. G. B. Olson, M. Cohen, Dislocations in Solids, F. N. R. Nabarro ed., Elsevier Science Publishers, 1986, 297-407.

24. E. Patoor, M. Berveiller, Mechanics of Solids with Phase Changes, CISM 368, M. Berveiller, F. D. Fischer eds., Springer Wien-New-York, 1997, 121-188.

25. V. Randle, The Measurement of Grain Boundary Geometry, Institute of Physics Publ., Bristol and Philadelphia, 1993.

26. B. Raniecki, K. Tanaka, Int. J. Engng. Sci., 32, (1994) 1845-1858.

27. A. L. Roitburd, Solid State Physics 33, (1978) 317-390.

28. J. C. Slattery, Interfacial Transport Phenomena, Springer-Verlag, New York, 1990.

29. M. J. Stowell, Epitaxial Growth. Part B, J. W. Matthews ed., Academic Press, 1975, 437-492.

30. A. Vivet, C. Lexcellent, J. Phys. IV France 9, (1999) Pr9-411-418.

31. T. Y. Thomas, J. Math and Mech., 6, (1957) 311-322. 